\title{
Analyses du Développement et Théories de la Dependance en Amérique Latine. L'actualite d'un débat.
}

\author{
Robert Rollinat
}

Resumo: 0 objetivo principal deste artigo consiste em avaliar em que medida as teorias da dependência, particularmente aquelas ligadas a CEPAL, poderiam servir de referência para contribuir, em uma perspectiva crítica, para um melhor entendimento do atual debate sobre o desenvolvimento da América Latina.

Palavras-chave: Desenvolvimento, Teorias da Dependência, América Latina.

Résumé: L'objectif principal de ce texte est d'évaluer dans quelle mesure les théories de la dépendance, en particulier celles liées à la CEPAL, pourraient servir de référence pour contribuer, dans une perspective critique, à une meilleur compréhension du débat actuel sur le développement de l'Amerique latine.

Mots-clés: Developpement, Theories de la Dependance, Amerique Latine.

"Professeur d'Economie FORUM-DET, Université de Paris 10-Nanterre. GREITD.E-mail: rrollinat@wanadoo.fr 
"La ùnica razòn, la ùnica excusa para estudiar la teorìa économica es para hacer del mundo un mejor lugar en donde vivir"

W.C. Mitchell

\section{Introduction}

Qu'est-ce qui justifie aujourd'hui que l'on reprenne aujourd'hui, en Amérique latine, les discussions sur le thème de l'impérialisme et de la dépendance?

i) Tout d'abord, l'évolution même de la situation économique et géopolitique du souscontinent où les questions de la dépendance et de la domination économique n'ont jamais, après maintenant près de vingt ans de mise en œuvre des politiques néolibérales, été vécues avec autant d'acuité.

ii) Le constat que, si beaucoup des anciens auteurs et adeptes de la Théorie de la Dépendance ont abandonné ou remis en question ses fondements analytiques, d'autres auteurs y attachent encore un certain crédit ${ }^{1}$

iii) La troisième raison: la situation même de la recherche en matière de développement dont le paradigme serait entré en crise et même, pour certains, en complète désuétude.

Un des objectifs de ce texte sera donc d'évaluer dans quelle mesure les théories de la dépendance pourraient servir de référence pour contribuer, dans une perspective critique, à un enrichissement ou un renouveau des analyses actuelles du développement.

Pour tenter de cerner, en Amérique latine, les fondements de cette Théorie de la Dépendance avec la domination économique et politique qu'elle suppose, il est d'abord nécessaire de rappeler et de tirer un rapide bilan des analyses de la CEPAL en matière de développement.

\section{Le Developpement Modernisateur de la CEPAL: le "Desarrollismo"}

C'est à l'issue de la seconde guerre mondiale que, sous l'égide de l'ONU, vont s'affirmer les conceptions "développementistes" de la Commission Economique pour l'Amérique Latine. Pour la CEPAL, sous l'influence des théories de la croissance "par

${ }^{1}$ Il est, de ce point de vue, significatif que, dans le " best-seller " culte du libéralisme et de la fin des idéologies, " $\underline{\boldsymbol{L} \boldsymbol{a}}$ fin de l'bistoire et le dernier bomme ", Fukuyama consacre un chapitre entier (chap.9) à une féroce critique de la théorie de la dépendance, considérée comme "la plus récente tentative de conserver une forme de marxisme dans le Tiers Monde" (FUKUYAMA, 1992, Flammarion) 
étapes", le développement est conçu comme un processus évolutifcontinu. Ce continuum n'oppose donc pas “développement" et "sous-développement". Celui-ci n'est qu'une étape antérieure de celui-là et le développement doit être accessible à tous les pays qui s'engageront à en créer les conditions adéquates (MARINI , 1993, p. 60).

Parmi ces conditions, la CEPAL met l'accent sur la nécessaire "modernisation", au plan économique, social, institutionnel, idéologique, des structures "retardées" des pays de la "périphérie" afin de combler l'écart avec les pays du "centre".

Bien que les conceptions cépaliennes puissent s’appliquer, à l'issue de la guerre, à tous les pays du Tiers Monde, notamment ceux qui s'engagent, en Afrique ou en Asie, vers le rejet des empires coloniaux européens, les premiers travaux initiés en 1948 à Santiago du Chili vont plus spécialement s'appliquer à la réalité latino-américaine.

En effet, les sociétés d'Amérique latine paraissent en effet tout à fait représentatives de ce "dualisme structurel" qu'il faudrait surmonter. Ce ne sont plus des pays "neufs": l'indépendance politique y est déjà ancienne (déjà plus d'un siècle), les Etats nationaux s'y sont consolidés. Mais la structure des classes, complexe, est restée très inégalitaire. Le "desarrollismo" latino-américain, très influencé par le keynésianisme, apparait donc comme une théorie du développement tout à fait adaptée à un sous-continent dont certains pays avaient commencé à s' industrialiser dans l'entre-deux guerres.

Un des apports théoriques essentiels de la doctrine cépalienne, c'est la critique classique de la théorie du commerce international fondée sur le principe des avantages comparatifs et selon lequel chaque pays doit se spécialiser dans les biens où, grâce à sa productivité, il aura un avantage par rapport à ses concurrents. C'est en suivant ce principe que tous les pays pourraient gagner à l'échange. La CEPAL montre que, dans les faits, ce principe ne s'est pas appliqué à l'Amérique latine. Depuis 1870, les termes de l'échange des matières premières exportées par les pays latino-américains n’ont cessé de se détériorer.

C'est un processus qui engendre mécaniquement la dépendance vis-à-vis de l'extérieur. Pour échapper à cette contrainte internationale sur les prix des biens primaires et à cette situation structurellement inégalitaire, il est donc nécessaire de promouvoir une industrialisation interne afin de produire des biens manufacturés pour la demande intérieure. Il faut produire dans la "périphérie" ce qui était jusque là importé du "centre", mettre en œuvre une politique d'industrialisation par substitution d'importations (ISI). C'est

\footnotetext{
Celles en particulier développées par W.W.Rostow ("The Process of Economic Growth", 1953) et W.A. LEWIS ("The Theory of Economic Growth", 1955 mais aussi J. B. Clark (Conditions of Economic Progress", 1951) pour l'analyse du rôle respectif des différents secteurs (primaire, secondaire, tertiaire) dans le développement.
} 
en particulier par un accroissement de la productivité interne que l'on pourra abaisser le prix des biens produits. "L'industrialisation à la périphérie" aura aussi pour conséquence de corriger les déséquilibres et les inégalités sociales dans les pays concernés.

Laccent mis par la CEPAL sur les vertus magiques de l'industrialisation doit permettre d'élever le niveau de vie des masses populaires sans que soit par exemple nécessaire une politique de "redistribution des revenus", ou même une réforme agraire .

On pourrait ainsi considérer que le "desarrollismo" représente, en Amérique latine, les intérêts et l'idéologie d'une bourgeoisie "industrielle nationale" soucieuse de s'affirmer face à une bourgeoisie " exportatrice " essentiellement attachée à réaliser les gains issus du commerce des biens primaires et souvent alliée aux oligarchies foncières et aux intérêts étrangers. Assez naturellement, dans le cadre national, cette "bourgeoisie" industrielle en développement pourra être conduite à s'allier aux ouvriers d'industrie et à la classe moyenne salariée.

Une question peut ici être posée: pourquoi les Etats-Unis ont-ils, à l'issue de la guerre, "toléré" la CEPAL comme institution "indépendante", alors que normalement elle devait être intégrée, comme commission spécialisée, à l'OEA (Organisation des Etats Américains)? C'est certainement une contrepartie aux décisions de la Conférence de Bretton Woods où les latino-américains, (dont la plupart avaient défendu le "plan Keynes") n'avaient guère été entendus. Il apparaît aussi difficile, à l'issue de la seconde guerre mondiale, de neutraliser un courant nationaliste industrialisateur particulièrement actif en Amérique latine, en particulier au Mexique et au Brésil. Mieux valait peut-être tenter d'assurer son contrôle de l'intérieur ${ }^{4}$. Le rôle politique de ces deux pays semble en tout cas avoir été déterminant dans cette décision.

\section{Crise du "Desarrollismo" et Conceptions Dependantistes}

Les critiques de la vision cépalienne ont eu beau jeu de critiquer une approche privilégiant les conflits de nations (considérées comme homogènes), donc oublieuse des classes sociales, ainsi qu'une approche des rapports internationaux trop "économiciste"

\footnotetext{
${ }^{3}$ Ainsi, pour Prebisch, le problème essentiel de l'Amérique latine, c'est la hausse du Revenu réel par tête grâce àl'accroissement de la productivité puisque, selon lui, les instruments de la redistribution n'ont qu'une portée très limitée (PREBISCH, 1986).

¿4 Lorsque qu'en 1951, à l'ONU, la question de la remise en cause de l'indépendance institutionnelle de la CEPAL sera posée pour officiellement en "réduire le coût de fonctionnement", ses membres recevront le soutien du Brésil et en particulier du Président Getulio VARGAS parfois considéré comme "le père de l'industrialisation brésilienne". La démarche effectuée pour le remercier par Raùl Prebisch et Celso Furtado est racontée dans (FURTADO, 1987).
} 
car presque exclusivement fondée sur la prise en compte des mécanismes de l'échange et de la balance des paiements. L'Etat y est en outre considéré comme une institution socialement neutre susceptible d'adopter des lois et des mesures économiquement rationnelles, dans l'intérêt national.

On va assister assez rapidement à une crise du "desarrollismo" incarné par la CEPAL (O'BRIEN; ABEL, LEWIS, 1985). Dès le début des années 1960, la CEPAL tente d'élargir sa vision en insistant sur la nécessité des réformes structurelles dans le processus de développement . Mais il est déjà trop tard car le cycle des dictatures militaires est engagé (BRÉSIL, 1964) et certains de ses initiateurs sont déjà contraints de s'exiler.

Prebisch a rejoint l'UNCTAD et Celso Furtado écrira en 1965 "Développement et sous-développement" qui, dit-il concerne les économies, qui ont épuisé la possibilité de la substitution d'importations". Il montre alors que la tendance à la stagnation des économies latino-américaines découle, non pas tant de la mise en œuvre de telle ou telle politique économique (ce qui absout la CEPAL) mais résulte des structures économiques de la région. (FURTADO, 1965).

Si l'on ne peut pas considérer que la théorie de la dépendance constitue une extension "radicalisée" des conceptions de la CEPAL car les fondements théoriques sont différents, il est certain qu'elles en constituent le prolongement.

Marini rappelle qu'une des origines de la théorie de la dépendance ce sont des auteurs qui, dans l'entre deux-guerres ont mis l'accent sur 'l'identité et la spécificité latino-américaines" comme le péruvien Mariategui et aussi la gauche latino-américaine de l'entre-deux guerres, notamment celles des Partis Communistes, même s'ils sont en Amérique Latine faiblement implantés. Ce sont les conceptions de la $3{ }^{\text {ème }}$ Internationale pour laquelle l'Amérique Latine est identique à l'Asie (on la qualifie même de "Chine de l'Extrême Occident"). Donc, les thèses de l'Internationale Communiste sur la question coloniale doivent s'y appliquer (MARINI, 1993).

La perspective politique serait alors la Révolution démocratique bourgeoise, antiféodale et anti-impérialiste, qui ne pourra être réalisée qu'en postulant l'existence d'une bourgeoisie nationale susceptible de la mener à bien. Il est évident que pour ce front anti-impérialiste supposant l'alliance de la bourgeoisie et des classes populaires, le "gradualisme réformiste" de la CEPAL était aussi tout à fait convenable.

Cependant, en Amérique latine, dans les années 50, une gauche non "communiste soviétique" a émergé, assez composite, parfois issue des mouvements sociaux. Elle va constituer avec un certains nombre d'auteurs en Amérique latine et à l'étranger, le support des nouvelles conceptions. La vision la plus "radicale" du dépendentisme des origines est due à Gunder Frank. 
C'est en s'appuyant sur l'évolution historique concrète de deux pays latino-américains depuis l'Indépendance, le Chili et le Brésil, qu'André Gunder Frank avait pu poser les bases de son analyse du "développement du sous-développement". Le point de départ de sa démonstration, contrairement à la CEPAL, est que :

- le sous-développement n'est pas du à la survivance d'institutions archaïques ou à l'existence d'une "pénurie de capital" dans les régions isolées des courants mondiaux d'échange mais est et demeure engendré par le même processus qui a généré le développement du capitalisme lui-même (GUNDER FRANK, 1967, 1969).

- donc, conséquence : pour les pays "dépendants", participer comme "satellite" aux échanges mondiaux conduit nécessairement à une structure économique dépendante et déséquilibrée, à un rapport d'exploitation marqué par un transfert contraint des surplus réalisés.

La théorie de la dépendance tend donc à considérer le capitalisme comme un "système mondial " et rejette la conception "évolutionniste" selon laquelle développement et sous-développement seraient des phases distinctes d'un même processus. Ce sont au contraire des réalités opposées. La (relative) prospérité des pays du "centre" ne peut se faire qu’au détriment des pays de la "périphérie". L'impérialisme n’est pas "extérieur" au développement, au contraire, il imprègne toute l'économie de la société dépendante, représentant un facteur essentiel dans la constitution de sa structure socio-économique, de son Etat, de sa culture (BAMBIRRA, 1978).

C'est pourquoi, dans sa version "radicale", la "dépendance" ne peut être surmontée dans le cadre du capitalisme. La lutte pour la dépendance ne peut donc être dissociée de la lutte pour le socialisme.

L'analyse de Gunder Frank trouve son prolongement dans l'analyse qu'il fait des relations entre les mécanismes de la dépendance et la caractérisation des classes sociales, et ce depuis la conquête coloniale des Amériques. En Amérique latine, l'émergence d'une "lumpenbourgeoisie" a été consubstantielle au lumpen-desarrollo (une forme de développement frustré, hybride). Une classe compradore subordonnée marque la dépendance coloniale liée à l'expansion du capitalisme commercial européen et c'est cette relation qui perdure.

En soulignant, pour leur part, que les formes de la dépendance peuvent varier considérablement, Cardoso et Faletto insistent sur le caractère "historico-structural " de cette dépendance, ce qui doit permettre de repérer, notamment en Amérique latine, les transformations importantes . Au niveau économique, un pays doit être considéré

\footnotetext{
Ce constat conduit à une différenciation inégalitaire entre des métropoles "dominantes " et leurs satellites "dominés" comme l'atteste l'évolution de nombreux pays d'Amérique latine. Une des conséquences de cette approche est que les "satellites" connaissent leur développement économique le plus fort lorsque les liens avec les
} 
comme dépendant "lorsque l'accumulation et l'expansion du capital ne peuvent trouver leurs composantes dynamiques à l'intérieur même du système" (CARDOSO, FALETTO, 1978, p.20-21). Les économies "périphériques", même lorsqu’elles ne sont plus simplement productrices de matières premières, connaissent alors une forme bien particulière de dépendance: leur production de biens capitaux est insuffisante pour assurer une progression continue du système, "aussi bien sur le plan technologique et financier qu'en termes organisationnels"(p.22).

Mais Cardoso et Faletto rejettent toute "théorie" globale du capitalisme dépendant. Ils insistent surtout sur des situations de dépendance déterminées par les étapes de l'expansion, à l'échelle mondiale, du capitalisme lui-même.

Un certain nombre de critiques, notamment d'un point de vue marxiste, ont été faites à la théorie de la dépendance. Ont été en particulier dénoncées certaines insuffisances de l'analyse (MATHIAS, SALAMA, 1993).

- une vision simplificatrice et statique de l'économie mondiale, considérée comme une donnée peu susceptible de contradictions.

- une vision trop réductrice du capital, considéré comme simple rapport technique de production indépendamment des rapports sociaux,

- des Etats trop mal définis du point de vue des classes sociales conduisant à des situations

"d'industrialisation restreinte" ou "retardataire" au statut mal défini

- un pouvoir politique trop "dépendant" de l'économique . On a pu ainsi reprocher à la théorie dépendandiste de théoriser une forme " d'Etat populiste ", fondé sur un "pacte" politique entrel'oligarchie terrienne, une bourgeoisie et un prolétariat industriel naissants.

On a aussi parfois considéré la théorie de la dépendance comme l'expression d'un “nationalisme romantique" dans la mesure où elle tend, de façon parfois idyllique, à

\footnotetext{
"métropoles" se distendent. Les phases historiques où l'Amérique latine aurait connu son plus grand essor, notamment sur le plan industriel, correspondraient ainsi à des périodes où la pression du "centre" sur la "périphérie" se serait fortement atténuée: dépression espagnole du XVIIème siècle, guerres napoléoniennes, première guerre mondiale, crise mondiale des années 1930, second conflit mondial. Cette conclusion s'oppose à la thèse généralement admise selon laquelle la croissance des pays sous-développés découlerait d'une intégration plus poussée avec les pays du "centre" (STAVENHAGEN, 1973).

La relecture de la préface de l'édition anglaise de 1979 de Cardoso-Faletto invalide, nous semble t'il, cette critique: "Nous concevons la relation entre les forces internes et externes comme formant une totalité complexe dont les liens structurels ne sont pas basés sur de simples formes d'exploitation et de contrainte mais sont enracinées dans une conjonction d'intérêts entre les classes locales dominantes d'un côté et de l'autre sont contestées par les classes et les groupes dominés".
} 
occulter l'analyse de l'impérialisme (WARREN, 1980). Ainsi, il n'y aurait ainsi aucun espace "théorique" pour servir de support à l'étude concrète de cette dépendance qui apparaît ainsi comme un conecept flou, très largement récupérable par des différents courants de la société latino-américaine (BREWER, 1990). Pour d'autres critiques, la faiblesse des conceptions dépendantistes découlerait du fait qu’elles ne constituent pas un "programme" qui se caractériserait, par exemple, par une série de propositions concrètes de politique économique (PACKENHAM, 1992).

Les théories de la dépendance vont cependant largement modifier les approches du développement en Amérique latine car:

i) elles ont constitué un renversement complet de la littérature de "selfincrimination" qui consistait à se rendre soi-même responsable de l'impasse du développement. Hirschman avait évoqué, en la reprenant d'ailleurs partiellement à son compte, "la manie de l'échec" (la fracasomania) qui aurait été un des stigmates indélébiles de la société latino-américaine et notamment de ses élites.

ii) ces théories, dont on a souvent caricaturé les positions, n'impliquent pas seulement un conflit direct entre les nationaux ("l'intérieur") et l'étranger ("l'extérieur"), les bons et les méchants. Elle présuppose une coïncidence fondamentale d'intérêts entre les groupes dominants dans chaque type de société, sans évidemment nier la lutte de classes interne aux pays du Tiers Monde.

\section{Les Derives du Dependantisme: “Endogenismo" et Nouvelle Dependance}

En fait, l' évolution des conceptions dépendantistes va être assez largement tributaire des évènements politiques en Amérique latine. Après le coup d'Etat de Pinochet au Chili, certains des économistes "dépendantistes" qui s’y étaient réfugiés, doivent à nouveau s'exiler, notamment au Mexique. Bien qu'Allende n'ait guère eu le temps de mettre en pratique les conceptions dépendantistes, sa chute va cependant conduire à tirer un premier bilan.

Bien que ne pouvant prétendre représenter le mouvement dans son ensemble, le Congrès de San José de Costa Rica en 1974 va concrétiser le retour à un certain "marxisme historique", celui l'analyse des modes de production.

"L'endogenismo", le courant qui veut exprimer ce retour, reprend ainsi pour base explicative principale les modes de production et les formes d'accumulation originelle du capital dans les pays de la périphérie. Il pose comme hypothèse que le capitalisme latino-américain doit être considéré en lui-même et, dans un premier temps, indépen- 
damment de la question de l'impérialisme. L'impérialisme pourra être ensuite introduit mais "ex-post", une fois définie la spécificité de la formation sociale étudiée.

Il y a bien là un point de rupture avec la théorie initiale de la dépendance qui postulait que la constitution de l'économie capitaliste dépendante était inséparable du processus mondial qui engendre l'impérialisme .

La "nouvelle dépendance" prétend, elle aussi, opérer un retour sur le contexte et les formes de la croissance en Amérique latine. On a parlé de "néo-développementistes" (néo-desarrollistas) dans la mesure où, pour ses défenseurs, sont de nouveau discutées les conditions internes d'un développement autonome du capitalisme en Amérique latine.

Ainsi, dès 1973, Cardoso avait déjà quelque peu modifié son approche initiale de la théorie de la dépendance, donnant à la "nouvelle dépendance" un contenu bien spécifique, celui d'un modèle de "développement dépendant associ”. (modèle DDA). Quels sont les changements qui, selon lui, justifiaient désormais cette nouvelle approche ? Il convient ici de le citer intégralement:

"Les changements dans l'organisation capitaliste internationale ont produit une nouvelle division du travail. La force motrice de ce changement est la firme multinationale. Dans la mesure où elle intègre du capital industriel dans les économies périphériques, la nouvelle DIT met en action la dynamique interne du marché. Ainsi, jusqu’à un certain point, les intérêts des firmes étrangères deviennent compatibles avec la prospérité des pays dépendants. En ce sens, elles aident à promouvoir le développement. Pour cette raison, la croissance des FMN rend nécessaire une reformulation de la vision traditionnelle de l'impérialisme économique qui considère que la relation de base entre un pays développé et un pays sous-développé est une relation extractive d'exploitation qui perpétue la stagnation".. (CARDOSO, 1973; STEPAN, p. 149)

Cardoso considère désormais que la croissance économique générée par l’investissement massif de capital étranger bénéficiera essentiellement à certains secteurs, en particulier ceux des biens de consommation pour les classes moyennes et élevées de la société. Il estime aussi que ce développement "associè" sera basé sur un "profil régressif de distribution du revenu" fondé sur la consommation de biens de luxe de biens durables et non

7 Outre l'influence des "endogénéistas", deux autres évènements, politiques ceux-là, vont contribuer à la dérive et à la dispersion du dépendantisme : la formation du groupe "Contadora" (en réaction à la politique américaine en Amérique centrale) et en 1975, la fondation du SELA (Sistema Economico Latinoamericano) (duquel les Américains sont exclus) et qui va utiliser les services des anciens "développementistes": Prebish, Furtado, Cardoso, Pinto, Ferrer. 
pas de biens de première nécessité, ce qui aura pour effet d'augmenter l'endettement externe, de contribuer à l'exploitation des ressources en travail et à la marginalité sociale.

Se référant à l'exemple brésilien, cette nouvelle "donne" générera, selon Cardoso, une alliance entre les secteurs "anti-populistes" des militaires et le secteur international des affaires, ce qui aura pour effet d'affaiblir le nationalisme des pays dépendants. On verrait ainsi se développer une idéologie "militaire-managériale" dans le cadre d'un Etat qui ne serait pas totalitaire mais qui pourrait être considéré comme une "autocratie développementiste militaro-bureaucratique"(CARDOSO, 1973).

Il est certain que cette évolution théorique ne peut être dissociée de l'évolution politique de Cardoso lui-même, évolution qui le conduira, à l'issue d'une élection très controversée, à la Présidence du Brésil en 1994. Ce destin peu commun a évidemment contribué au discrédit des thèses dépendandistes .

Tous ceux qui, au Brésil, à l'instar de Cardoso, ont choisi la voie du pouvoir (LOUREIRO, 1997) ont logiquement rejeté le paradigme du structuralisme et de la dépendance qui s'est, selon Bresser Pereira, "révélé inopérant face aux difficultés des années 1980 et 1990". Rejetant également le "néo-libéralisme", ils préconisent désormais "une stratégie orientée vers le marché qui soit à la fois pragmatique et sociale-démocrate, ou socialelibérale, associant le renforcement de l'Etat et l'amélioration de la compétitivité économique au niveau international" (BRESSER PEREIRA, 1996, 2002).

Furtado et Prebisch n'entreront pas dans cette logique et continueront, au moins dans leurs écrits, à partir d'une vision globale de l'économie mondiale. Ils auront même parfois tendance, libérés de toute contrainte institutionnelle ou de gestion gouvernementale, à "radicaliser" leur discours antérieur.

Ainsi, Prebisch dans "Capitalismo periférico. Crisis y transformaciòn”, publié au Mexique en 1981, réaffirme t’il la validité de la distinction centre-périphérie. Tout en conservant sa spécificité, le capitalisme périphérique reste composante du système mondial (PREBISCH, 1981, p. 31). Il plaide pour une théorie globale du développement intégrant les phénomènes politiques et pas seulement la théorie économique.

\footnotetext{
${ }^{8}$ T. Dos Santos a relevé les incohérences du programme économique et social de Cardoso, sa " rupture fondamentale " avec le dépendantisme alors que, selon lui, la crise de la dette externe a renforcé la thèse fondamentale de la dépendance, les pays du centre ayant de fait institué, à leur profit, un "transfert négatif " expliqué en partie par un échange international inégal mais aussi par le gonflement du paiement de royalties, d'intérêts ainsi que le rapatriement des profits. Pour consolider la démocratie au Brésil, prendre de véritables mesures sociales, engager la réforme agraire et mettre en œuvre un changement dans les formes de la propriété, il était donc nécessaire de briser la dépendance et détruire le pouvoir hégémonique du capital monopoliste" (DOS SANTOS, 1998, p. 62). Dans un ouvrage postérieur, Dos Santos affirme cependant son accord de fond avec Cardoso sur la problématique de la "nouvelle dépendance" (DOS SANTOS, 2000, p. 123-130).
} 
Celso Furtado, pour sa part s'affirme de plus en plus sceptique quant aux possibilités du développement "autonome" des pays de la périphérie. Déjà en 1970 dans sa "Théorie du développement économique”, il avait analysé la tendance à la stagnation des économies "qui ont épuisé les possibilités de la substitution d'importations", ce qui avait déjà conduit à une forme "d'obstruction structurelle du développement". Il estimait nécessaire, pour surmonter cette situation, d'agir conjointement sur l'offre et la demande et de coordonner cette action. Cette coordination n'était, selon lui, possible que dans le cadre de la planification, "c'est-à-dire d'une stratégie capable de conditionner les processus économiques dans leur ensemble” (FURTADO, 1970, p. 214; p.226). Plus tard, il dénonce l'influence grandissante des sociétés multinationales:

"La prédominance de la logique des entreprises transnationales dans l'organisation des activités économiques conduira presque nécessairement à des tensions interrégionales, à l'exacerbation des rivalités corporatives et à la formation de grandes poches de misère, tout allant dans le sens de rendre non viable le pays en tant que projet national" (FURTADO, 1992, p. 35).

\section{Dependantisme et Structuralisme}

En Amérique latine, les théories dépendandistes ont donc largement influencé la sociologie et l'économie du développement. Cette influence intellectuelle considérable a largement débordé les frontières du sous-continent comme en témoignent les nombreuses traductions et rééditions des principaux ouvrages consacrés à la dépendance (et en particulier celui de Cardoso-Faletto à partir de l'édition originale espagnole de 1969). Le dépendantisme a inspiré un large éventail d'opinions : révolutionnaires, socialistes, nationalistes, religieuses (à travers notamment la théologie de la Libération), toutes liées à la transformation sociale et un changement politique. C'est cette influence durable qui a amené certains auteurs américains, à en critiquer les bases conceptuelles et aussi à tenter d'en "récupérer" certains aspects.

Ces économistes et sociologues, tout en reconnaissant son influence dans les institutions universitaires et de recherche ont remis en cause les fondements même de la théorie de la dépendance en critiquant le caractère abstrait et "utopique" de ses principales propositions.

Ils vont donc proposer de "mesurer" la dépendance des différents pays à partir d'un certain nombre de critères économiques ou par secteur d'activité. Dans la préface à la nouvelle édition anglaise de 1979 de "Dépendance et Développement", Cardoso et Faletto rejetteront cette conception purement fonctionnaliste, considérant qu'elle est totalement contradictoire avec la vision politique globale, à la fois structurelle et historique, de la Théorie de Dépendance. 
Packenham a proposé une distinction entre dépendance orthodoxe et dépendance hétérodoxe. Selon lui, la vision orthodoxe, c'est la vision purement holistique, celle qui opère le mélange entre marxisme et nationalisme (plutôt celle de Gunder Frank, Cardoso et Faletto "première manière" ou Dos Santos). La vision hétérodoxe, c'est celle qui pourrait justement faire l'objet de "propositions concrètes" (PACKENHAM, 1992) et donc fournir des éléments programmatiques aux "économistes de gouvernement". Pour ces derniers, la Théorie de la Dépendance doit désormais s'adapter aux "contraintes externes” et renoncer à toute forme de développement autonome désormais considéré comme utopique.

En fait, la théorie de la dépendance va l'objet d'un certain nombre de récupérations conceptuelles de la part d'auteurs comme Albert Hirschman par exemple où le terme de dépendance se définit désormais en termes de pouvoir d'influence. L'analyse de ce pouvoir pourra se faire grâce à des études empiriques autour des "bargaining studies" (analyses de "marchandage" de pouvoir).

Dans ces approches, on ne s'attache plus aux relations de domination économique avec tout ce que tout cela suppose en termes d'analyse des classes ou d'extorsion du "centre" sur la "périphérie" mais aux liens d'influence entre les multinationales, les Etatshôtes, les partis et les hommes politiques au pouvoir, etc. Beaucoup d'études empiriques intéressantes seront par exemple consacrées aux "marchandages" entre l'Etat et les firmes multinationales sur les cours internationaux des matières premières (celles de Krasner par exemple sur le café au Brésil) mais par la suite on étendra ces analyses aux relations générales de "conflit" entre pays du centre et de la "périphérie". C'est le sens notamment d'un ouvrage de Krasner sur le "conflit structurel” entre le "Tiers monde" et le libéralisme global”. Dans cette démarche, les conceptions dépendantistes, originelles sont édulcorées pour privilégier les "arbitrages" entre les intérêts contradictoires des différents pays (KRASNER, 1985).

Les conceptions liées au néostructuralisme vont, elles aussi, considérablement modifier l'approche structurelle initiale des théories de la dépendance. En effet, le cadre d'analyse de la CEPAL et des théoriciens dépendantistes s'appuyait sur la méthode “historico-structurelle" (les structures productives héritées du passé conditionnent la dynamique des économies latino-américaines et distinguent leur comportement de celle des pays développés) (BITAR, 1988).

Le néostruturalisme differe de ce structuralisme originel en ce qu'il se présente comme un instrument méthodologique d'une vision fonctionaliste des processus sociaux., le plus souvent détachée de l'histoire. Son champ d'analyse est d'ailleurs beaucoup plus restreint puisque pouvant par exemple s'appliquer en sociologie, en linguistique ou en anthropologie. L'analyse politique du développement va s'en trouver profondément modifiée . 


\section{Le Neostructuralisme et la Mondialisation}

French Davis a rappelé qu’il était difficile de définir avec précision les traits principaux du néostructuralisme, notamment comme alternative crédible à la pensée économique orthodoxe (FRENCH DAVIS, 1988). S'il est évident que le néo-structuralisme découle des conceptions structuralistes déjà évoquées (MOLERO, 1981), il reprend également toute une série de conceptions orthodoxes, au nom de la nécessaire adaptation à l'évolution économique et à "l'ouverture " de l'Amérique latine (PINTO, 1991).

Dans une formule condensée, les néo-structuralistes ont posé la caractéristique principale du "nouveau" développement latino-américain dans son rapport avec l'extérieur en rejetant l'ancien concept de "desarrollo hacia dentro" (développement pour (ou vers) l'intérieur) et en lui susbstituant le concept de "desarrollo desde dentro") (depuis (ou à partir) de l'intérieur) (SUNKEL, 1991). Derrière cette distinction sémantique, se met en place une nouvelle stratégie, non plus celle de "l'ancienne" industrialisation par substitution d'importations (I.S.I.) de la CEPAL mais des propositions qui, à partir du desarrollo desde dentro, vont permettre d'orienter l'industrialisation "vers certains marchés particuliers, internes ou externes". Ces marchés devraient désormais être prioritaires dans une stratégie de développement à long terme. Cette démarche prend comme hypothèse que les pays d'Amérique latine possèdent ou peuvent acquérir les bases technologiques et les "niveaux d'excellence relative" leur permettant de s'insérer solidement dans l'économie mondiale (SUNKEL, 1991; ZULETA, 1992).

Dans ce nouveau cadre, le processus d'industrialisation est centré sur l'offre (et non plus sur la demande interne comme dans la vision de la CEPAL). C'est cette offre qui devient essentielle et qui va déterminer 'l'accumulation, la qualité, la flexibilité, la combinaison et l'utilisation efficiente des ressources productives, l'incorporation du progrès technique, l'effort innovateur et la créativité.." (SUNKEL, 1991, p. 19). C'est aussi ce processus "d'innovation", très schumpeterien, qui dans la vision néostructuraliste, va influencer la capacité organisationnelle de la société, la discipline sociale, la frugalité de la consommation privée et publique ainsi que l'accent mis sur l'épargne nationale. Il s'agit bien d'un renversement du paradigme keynésien classique . Le nouveau développement

\footnotetext{
9 José Ocampo, dans un ouvrage consacré aux instruments à mettre en oeuvre pour stabiliser l'inflation en Amérique latine résume, à sa manière, un des aspects du néo-structuralisme: à la différence des phases antérieures de la pensée économique de la région, lorsque prévalait la démarche structuraliste classique, cette nouvelle école de pensée régionale, néo-structuraliste selon la dénomination de certains, a cherché à formaliser sa démarche conceptuelle en utilisant les instruments de l'analyse économique traditionnelle" (OCAMP0, 1990, p. 13).
} 
“industrialisateur" c'est un effort "depuis l'intérieur", c'est un développement qui peut s'entretenir de lui-même. Il faut donc promouvoir un effort créatif interne pour configurer une structure productive adaptées aux potentialités spécifiquement nationales et aussi tenter de tirer parti de "l'hétérogénéité structurelle "des sociétés latino-américaines.

Ramos rappelle aussi l'impératif "structurel" de développer l'industrialisation interne mais qui serait combiné avec la primauté d'une orientation "hacia afuera" (vers l'extérieur). Pour ce faire, il préconise l'utilisation d'instruments de politique économique actifs pour promouvoir, de manière sélective, les exportations (pas seulement en utilisant les droits de douane) et fixer un taux de change réel élevé et stable (RAMOS, 1991). Pour les néo-structuralistes latino-américains, c’est de cette manière qu'il serait possible de reprendre et de dépasser le défi "industrialisateur" originel de Prebisch et de générer un mécanisme endogène d'accumulation et de progrès technique permettant de retrouver une croissance "dynamique" par l'amélioration de la productivité (un concept-clef du vocabulaire néo-structuraliste). Les modèles qui, avant la grande crise asiatique des années 1997-1998, serviront de référence sont le Japon, les NPI (nouveaux pays industrialisés) que représentent les quatre "dragons" du sud-est asiatique mais aussi le Brésil.

Il faut cependant songer aux adaptations nécessaires pour que cette orientation soit mise en œuvre avec un "coût social" moindre. D’où la proposition de créer "un fonds de restructuration économique et de développement social" pour affronter les problèmes sociaux les plus difficiles. Il faut favoriser la "transformation productive "mais cette transformation doit s'opérer "avec équité" en tentant d'améliorer la distribution du revenu et en réduisant les situations de "pauvreté extrême" (LUSTIG, 1991).

Concernant la fonction économique de l'Etat, la vision néo-structuraliste a rompu avec la primauté, jusque là affirmée par la théorie de la dépendance, d'une institution utilisée au service des politiques sociales et de la redistribution pour en faire l'incontournable outil de "l'ajustement structure ", considéré comme inévitable. L'Etat doit donc contribuer à la transformation productive en favorisant un redéploiement du marché mais, dans le même temps, "minimiser les cồts de relocalisation de la main d'œuvre et l'impact des chocs externes sur les plus pauvres, tout en évitant une concentration excessive des revenus et de la richesse" (LUSTIG, 1991).

\footnotetext{
${ }^{10}$ L'analyse "concrète" de ce processus industrialisateur a certainement été le mieux développée par F. Fajnzylber qui a décrit les impératifs de "l'efficience" : une nouvelle structure productive, des relations institutionnelles entre la "planification" de l'Etat et le marché, des "alliances sociales" nouvelles capables de stimuler le "noyau endogène" de la nouvelle industrialisation. L'accent est également mis sur l'importance du "bloc agroindustriel" à la fois comme base d'un nouveau surplus et de l'autosuffisance alimentaire. (FAJNZYLBER, 1983, p. 278, 328).
} 
Est postulée la nécessité d'un "nouveau style" d'intervention étatique, sélectif, stratégique et complémentaire du marché, d'un Etat qui ne serait ni néo-libéral, ni interventionniste traditionnel mais un Etat démocratique et participatif ("concertador") (ROSALES, 1988), mais, dans un contexte d' ajustement qui privilégie délibérément les intérêts du marché au détriment de toute capacité, directe ou indirecte, de gestion étatique. La mise en garde, souvent réaffirmée, contre les "insuffisances et les failles du marché", base d'une doctrine qui se veut "hétérodoxe", relève d'un volontarisme assez naï, surtout dans un contexte où les privatisations et la globalisation des économies ont encore réduit le domaine d'intervention public.

Car, telle est bien la difficulté: la "transformation" productive doit préserver "les équilibres macroéconomiques de base" dans la mesure où le "développement soutenu" nécessite en particulier, la stabilité des prix, l'équilibre monétaire et fiscal (RAMOS, 1991; AMADEO, 1993).

\section{La CEPAL, la Banque Mondiale et le Paradigme Dependantiste}

La CEPAL va progressivement s'adapter à la vision "hétérodoxe" des néostructuralistes. Dans la mesure où elle n'a pu, à partir des années 1970-1980, vraiment influencer, directement ou indirectement, l'orientation économique et politique des pays d'Amérique latine, la CEPAL sera contrainte de répondre, à sa manière, $a u x$ nouveaux défis posés par la mondialisation. Elle va ainsi passer progressivement du paradigme " du développement dépendant " hérité de l'après guerre (avec l'Etat national comme agent supposé d'une politique économique active de substitution d'importation) à une vision plus large (à partir des années 1960) d'intégration régionale (RODRIGUEZ O., 2001). Elle considère que le renforcement de l'interdépendance intra-régionale doit fournir une base suffisamment large à l'accumulation et à la mise en œuvre du progrès technique .

A partir des années 1980, c'est le problème de la dette qui devient essentiel dans la mesure où les flux d'investissement étranger, stimulés par l'ouverture et la pression des firmes multinationales et des banques, ont conduit à un énorme surendettement. La CEPAL avance alors, au plan intérieur, la nécessité de considérer les coûts sociaux de l'ajustement en tenant compte de l'hétérogénéité structurelle des différents pays et, au

\footnotetext{
${ }^{11}$ C'est dans cette optique qu'il faut considérer la création de l'ALALC (Asociaciòn Latinoamericana de Libre Comercio) au traité de Montevideo en 1960 (qui donnera naissance vingt ans plus tard à l'ALADI: Asociaciòn Latinoamericana de Integraciòn). Entrent aussi dans ce cadre des institutions comme la Comunidad Andina (Pacte Andin) ou "El Mercado Comùn Centroamericano".
} 
plan externe, préconise des mécanismes de renégociation de la dette adaptés à chaque pays. Les politiques de privatisation d'actifs publics sont analysées mais sans que leur légitimité soit véritablement remise en question. Afin de surmonter la crise de la dette, la CEPAL préconise de promouvoir les exportations non traditionnelles sur le marché mondial afin de générer une source suffisante de devises (ZAPATA, 2001, p. 132).

Depuis lors, outre son travail d'analyse et d'expertise , la CEPAL a été confrontée aux crises et à la vulnérabilité régionale. Les formes brutales de la mondialisation avec une destruction importante du tissu industriel dans certains pays (Argentine, Brésil, Colombie, Mexique), les politiques de privatisation ont révélé la faible capacité des Etats à mettre en œuvre des politiques alternatives. Mais, il semble que désormais la nouvelle approche de l'institution soit davantage marquée par une fascination croissante envers les vertus de la concurrence et du marché que par l'action régulatrice, publique et sociale, de l'Etat ${ }^{12}$.

Aujourd'hui, à partir d'une "vision prospective pour le XXIème siècle", la CEPAL avance maintenant pour l'Amérique latine trois objectifs: le développement, l'équité et la citoyenneté ainsi que la nécessité d'une croissance économique soutenue comme "instrument d'équilibre”. Mais les moyens pour réaliser ces objectifs qui supposent la mise en place de "mécanismes de négociation sociale et politique" ne sont guère explicités. Tout au plus peut-on, suite aux conséquences désastreuses de la mise en place en Amérique latine des politiques néo-libérales, percevoir certaines inflexions du discours ${ }^{13}$.

Il est également intéressant pour nous d'analyser brièvement, du point de vue du paradigme de la dépendance, les propositions de la Banque mondiale. Ses propositions de politique économique dans les pays en voie de développement semblent avoir évolué de la mise en valeur néo-libérale de l'Etat minimum, symbolisé par le Consensus de Washington, vers une perspective qui plaide en faveur d'un Etat plus actifet efficient.

En effet, dans les années 1980 et 1990, la Banque Mondiale a encouragé la libéralisation du commerce et de l'investissement, la dérégulation des marchés de capitaux, la discipline fiscale et une réduction de la dimension et du rôle de l'Etat par le biais de la privatisation des entreprises publiques ainsi que de brutales baisses des dépenses sociales.

\footnotetext{
${ }^{12}$ Ainsi, dans un document de travail de 1996: "Fortalecer el desarrollo. Interactiones entre macro y microeconomia": "l'affirmation de la concurrence est cruciale, aussi bien pour le développement productif et la compétition internationale que pour le bien-être des consommateurs".

${ }^{13}$ Ainsi,J. A. Ocampo dans un important document de 1999: "Sin dejar de lado la estabilidad macroeconomica $y$ las ventajas de contar con mercados màs abiertos, el mejor funcionamiento de dichos mercados.se visualiza cada vez màs como un instrumento complementario para buscar objectivos que, màs allà del crecimiento econòmico, incluyan también la equidad y la cohesiòn social, la sostenibilidad ambiental y el desarrollo democràtico". (OCAMP0, 1999).
} 
Aujourd'hui, il semblerait que la Banque mondiale ait "découvert" l'importance de l'Etat et des institutions qui, désormais, "comptent pour le développement". Elle a donc commencé à préconiser la mise en place d'institutions susceptibles de promouvoir l'efficacité économique et la croissance. D’où des réformes de “seconde génération” qui devraient venir compléter le programme néo-libéral initial (BURKI, PERRY, 1998):

- Renforcement des systèmes judiciaires

- Mise en place de régulations bancaires et des marchés de capitaux

- Combat contre la corruption et pour "l'efficience" de l'Administration

- Décentralisation du pouvoir politique, fiscal et administratif trop centralisé avec des niveaux "intermédiaires" de gouvernance.

L'origine de cette inflexion est due aux protestations consécutives aux désastres occasionnés par les politiques d'ajustement structurel qui, de l'aveu même de la Banque, n’ont pas réduit la pauvreté, ni relancél'investissement (TEICHMAN J., 2004). L'expérience de la chute des régimes soviétiques en Europe de l'Est et aussi la crise asiatique ont révélé que l'absence de toute régulation conduisait parfois à des situations de chaos économique et politique. D'où, l'idée de mettre en place de "bonnes institutions" pour promouvoir le marché (surtout en Amérique Latine où le retard institutionnel très important). Des règles claires et des institutions seraient donc désormais nécessaires pour faire respecter les droits de propriété et les mécanismes du marché dont la logique reste cependant primordiale.

Bien loin des objectifs initiaux des théories de la dépendance, on aboutirait ainsi à une version consensuelle commune "aseptisée" des formes du développement latinoaméricain. La mondialisation étant considérée comme incontournable, le "retour" à l'Etat et aux institutions n'aurait d'autre fonction que de "moduler" les effets destructeurs de la concurrence et du marché, notamment pour les plus démunis, afin de préserver l'équilibre précaire des sociétés (APTER, 1988; PETRAS, MORLEY, 1990).

\section{Politique et Economie en Amerique Latine: L'Actualite du Dependantisme}

Pourtant, un très rapide bilan de "l'actualitê" de l'économie latino-américaine tend à confirmer les conceptions d'ensemble de la "vieille " théorie de la Dépendance:

i) au niveau de la détérioration de termes de l'échange:

Cela est facilement perceptible quand on analyse, par exemple, l'écart moyen des revenus. En 1965, 52 des 102 pays du Tiers Monde non producteurs de pétrole 
entraient dans le quintile des plus bas revenus. Ils sont aujourd'hui 92. Les termes de l'échange n'ont cessé de se dégrader : selon la CNUCED, une baisse de $50 \%$ entre fin des années 1990 par rapport à 1970 . Entre 1980 et 1990, le cours moyen des matières premières ont baissé de $45 \%$.

ii) concernant la reprimarisation de l'économie. L'embellie actuelle, toute relative, des exportations, est essentiellement due à des secteurs à faible valeur ajoutée : le pétrole, le café, le soja (le Brésil : 31\% du marché mondial en 2003). Le défi “industrialiste” de la "vieille" Cepal et des dépendentistes n’a jamais autant paru d'actualité.

iii) quant aux revenus de l'émigration. La contrainte de l'émigration fait aujourd'hui, partiellement, de l'Amérique latine une économie de rente. En 2003, 38 milliards de dollars ont été envoyés de l'extérieur en Amérique centrale, au Mexique, au Brésil, soit un montant bien supérieur aux investissements étrangers.

iv) quant au montant de la dette externe, encore et toujours. Mais jamais peut-être les contraintes qui ont pesé sur son remboursement n'ont paru aussi fortes. L'exemple de l'Argentine est particulièrement illustratif. Ce pays est menacé de procès en saisie par le FMI et les créanciers internationaux privés de ses avoirs financiers et physiques à l'extérieur mais aussi contrainte de mettre en œuvre, au plan intérieur, les mesures dictées par les “money doctors” (DRAKE, 1994): réforme de la gestion de l'Etat et des provinces, recapitalisation des banques, fixation des tarifs des quelques services publics encore existants.

D’autres exemples pourraient être cités : les stratégies d' investissement et de placement financier de capitaux étrangers, la politique des firmes et des banques multinationales, le rapatriement des bénéfices, etc.

Quelles sont alors les analyses explicatives ou les mesures de politique économique applicables à l'Amérique latine qui pourraient aujourd'hui s'inspirer le " modèle" de la dépendance?

i) La nécessité d'approfondir l'analyse du caractère "dépendant" des élites latinoaméricaines, au plan politique, idéologique, économique non seulement vis-à-vis des Etats de "centre" mais aussi des firmes multinationales de production et de banque afin de contribuer à la mise en place d'un "Etat impartial" au service des citoyens et des intérêts publics et sociaux.

ii) L'exigence impérative d'un financement interne et "territorialisé" du développement économique ce qui suppose le développement, pour favoriser le crédit aux entreprises, de Banques d'Investissement Publiques Spécialisées capables, grâce à des mesures fiscales appropriées, de drainer l'épargne locale et de faire du réescompte (ce qui est 
contradictoire avec la dépendance financière et le caractère extraverti des financements "contraints" ou liés des banques privées internationales sous l'égide du FMI) .

iii) La reconquête de la souveraineté monétaire avec ses attributs "naturels": une Banque centrale non pas "indépendante" mais sous contrôle démocratique de l'Etat, capable de maîtriser l'émission monétaire, les taux d'intérêt, de décider du cours de la monnaie et du change. Cette politique de la monnaie et du change s'oppose radicalement à la "dollarisation" des économies et aux politiques de convertibilité forcée.

En matière monétaire et bancaire, les travaux de Raùl PREBISCH inspirés certes d'un contexte historique différent, constitueront une référence particulièrement utile (PREBISCH, 1986; PAZOS, 1988; SPROUT, 1992).

iv) La réhabilitation de la notion de service public et d'entreprise publique comme moyen pour l'Etat d'orienter une part de la production nationale et d'influencer la politique des prix. Les contrats de concession doivent en particulier définir, grâce à un cadre juridique approprié, l'articulation des intérêts publics et privés.

v) Un contrôle différencié des cours du change doit permettre de définir une hiérarchie quant aux besoins prioritaires en matière d'importations et permettre le contrôle des flux de capitaux, évitant leur fuite et la spéculation (FURTADO, 1987; PREBISCH,1990).

Ces quelques propositions de politique économique (qui mériteraient chacune un développement spécifique) ne sont évidemment pas envisageables en dehors d'une volonté politique de reconquête des fonctions sociales, monétaires, fiscales de l'Etat (nécessité en particulier d'une véritable réforme fiscale afin de lui procurer les ressources nécessaires). Elles constituent cependant les bases concrètes d'un possible nouveau paradigme du développement et du retour à la croissance.

D'aucuns pourront considérer avec scepticisme la mise en œuvre d'un tel programme. Hirschman prétendait qu'il y avait un style de gouvernement typiquement latino-américain car, disait-il, de nombreux problèmes ne sont pas traités en raison d'un "déficit d'intermédiation", ce qui fait que les détenteurs du pouvoir n'en ont pas connaissance. Les problèmes ne devenaient, disait-il, visibles que lorsque le niveau de mobilisation de la société atteignait un degré tellement élevé qu'un traitement de l'urgence était nécessaire pour désamorcer une situation pouvant aller jusqu'à la révolte.

C'est précisément dans ce type de conjoncture que certains pays d'Amérique Latine sont déjà entrés et c'est à cette situation qu'il faut aujourd'hui tenter de répondre. 


\section{Bibliographie}

ABEL, C., LEWIS C. M. (Eds.). Latin America, economic imperialism and the state: the political economy of the external connection from the independance to the present. Londres: Athlone Press, 1985.

AMADEO, E. J. Estabilizacion y reforma estructural en América Latina. Bogotà: TM Editores, 1993.

APTER, D. E. Rethinking development, modernization, dependency and postmodern politics. Sage, 1988.

BAMBIRRA, V. El capitalismo dependiente latino-américano. Mexico: Siglo 21 Editores, 1985.

BITAR, S. Neo-conservatism vs neostructuralism en America Latina. CEPAL Review, Santiago du Chili, n. 34, p. 45-62, april. 1988.

BRESSER PEREIRA, L. C. Crise et réforme de l'Etat au Brésil. Pour une nouvelle interprétation de l'Amérique latine. Traduit du portugais (1996). Paris: Editions de la MSH, 2002.

BREWER, A. Marxist theories of imperialism: a critical survey. London: Routledge, 1990. New York, 1ère éd., 1980.

BURKI, S. J., PERRY G. E. Beyond the Washington consensus: Institutions Matter. Washington: World Bank, 1998.

CARDOSO, F. H. Associated-dependant development: theorical and practical implications In: SPEPAN A. (Ed.). Authoritarian Brazil. Yale University Press, 1973, p.142-175.

CARDOSO, F. H.; FALETTO E. Dépendance et développement en Amérique Latine. (traduit de l'espagnol, Siglo 21, 1969). Paris: PUF, 1978.

DOS SANTOS, T. The theorical foundations of the Cardoso government. A new stage of the dependency-theory debate. Latin American Perspectives, v. 25, n. 1, p. 5370, january. 1998.

DOS SANTOS, T. A Teoria da dependência: balanço e perspectivas. Rio de Janeiro: Civilização Brasileira, 2000.

DRAKE, Paul. (Ed.) Money doctors, foreign debts and economic reforms in Latin America from the 1890s to the present. Jaguar Books on Latin America. Wilmington, 1994.

FAJNZYLBER, F. La industrializaciòn trunca de América Latina. CET, Nueva Imagen. Mexico, 1983.

FURTADO, Celso. Dialectica del desarrollo. Mexico: Fondo de Cultura Economica. 1965.

FURTADO, Celso. Théorie du développementéconomique. PUF. Traduit du portugais, 1970.

FURTADO, Celso. La fantaisie organisée: le développement est-il encore possible? Traduit du portugais. Paris: Publisud, 1987. 
FURTADO, Celso. Brasil: a construção interrompida. São Paulo: Paz e Terra. 1992.

GUNDER FRANK, A. Capitalism and underdevelopment in Latin America. Historical studies of Chile and Brazil. Monthly Review Press, New York, 1967.

GUNDER FRANK, A. The Development of underdevelopment. Monthly Review Press, New York, 1969.

GUNDER FRANK, A. Lumpen-burguesia: Lumpen-desarrollo. Dependancia, clase y politica en Latina América. Buenos-Aires: Ed. Periferia, 1973.

KRASNER, S. Structural conflict: the third world against global liberalism. University of California Press, 1985.

LOUREIRO, M. R. Os economistas no governo. Rio de Janeiro: Fundacion Vargas, 1997.

LUSTIG, Nora. Equidady desarrollo. In SUNKEL, 1991.

MARINI, Mauro R. Dialéctica de la dependancia: la economia exportador. In: Tres ensayos sobre America Latina. Barcelona: Ed. Anagrama, 1973, p. 91-135.

MARINI Mauro R. América Latina: integracion y democratia? Caracas: Ed. Nueva Sociedad, 1993.

MATHIAS G.; SALAMA P. L'Etat surdéveloppé. Des métropoles au tiers monde. La Découverte. Paris: 1983.

MOLERO, J. (Comp.). El analisis estructural en economia : ensayos de América Latina y España . Mexico, Madrid: Fondo de Cultura Economica, 1981.

OCAMPO, J. A. Inflaciòn y estabilizaciòn en América Latina. Nuevos modelos estructuralista. Bogotà: Tercer Mundo Editores, 1990.

OCAMPO, J. A. Màs allà del Consenso de Washington: una visiòn desde la CEPAL. Revista de la CEPAL, Santiago du Chili, n. 66, 1999.

PETRAS, J. (Ed.). Latin America: from dependance to revolution? 1973.

PACKENHAM, R. A. The dependency movement. Harvard University Press, London: Cambridge, 1992.

PAZOS, F. Raùl Prebish, Central Banker. CEPAL Review, Santiago, n. 34, p. 179-195, april, 1988.

PETRAS, J.; MORLEY, M. US Hegemony under Siege: class, politics and development in Latin America. London, New York, Verso, 1990.

PINTO, A. América Latina: una visiòn estructuralista. Mexico: UNAM, 1991.

PREBISCH, R. Obras: 1919-1948. Buenos Aires: Fundacion R. Prebisch, 1991.

PREBISCH, R. La crisis del desarrollo argentino. De la frustraciòn al crecimineto vigoroso. Buenos Aires: 1986.

RAMOS, J. Equilibrios macroeconomicos y desarrollo. In: SUNKEL, 1991. 
RODRIGUEZ, O. Fundamentos del estructuralismo latino-americano. Comercio Exterior, Mexico, v. 51, n. 2, febrero. 2001.

ROSALES, O. El neostructuralismo en América Latina. Pensamiento Iberiamericano, Madrid, n. 14, p. 369-373, julio-dic. 1988a.

ROSALES, O. An assessment of the structuralist paradigm for Latin American development and the prospects for its renovation. CEPAL Review, n. 34, april, 1988b.

SPROUT. The Ideas of Prebish. CEPAL Review, Santiago, n. 46, p.177-192, april, 1992.

STAVENHAGEN, R. Sept thèses erronées sur l'Amérique latine ou comment décoloniser les

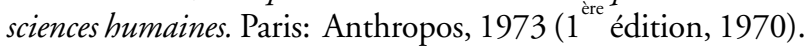

SUNKEL, O. El desarrollo desde dentro. Un enfoque neostructuralista para la América Latina. Mexico: Fondo de Cultura Economica. 1991.

TEICHMAN, J. The World Bank and policy reform in Mexico and Argentina. Latin American Politics And Society, Miami, v. 46, n. 1, p.39-74, spring. 2004.

WARREN, Bill. Imperialism, pionner of capitalism. Londres: 1980.

ZAPATA, M. R. Evoluciòn de las propuestas de la CEPAL: su aporte al desarrollo. Comercio Exterior, Mexico, v. 51, n. 2, febrero. 2001.

ZULETA, G. El desarrollo desde dentro: un enfoque neoestructuralista para América Latina"(Reseña). Pensamiento Iberoamericano, Madrid, n. 21, p. 304-313, enero-junio. 